Meta

Journal des traducteurs

Translators' Journal

\title{
De la nécessité pour le traducteur de maîtriser les domaines spécialisés : le cas des systèmes juridictionnels
}

\section{Héba Medhat-Lecocq}

Volume 55, numéro 2, juin 2010

URI : https://id.erudit.org/iderudit/044238ar

DOI : https://doi.org/10.7202/044238ar

Aller au sommaire du numéro

Éditeur(s)

Les Presses de l'Université de Montréal

ISSN

0026-0452 (imprimé)

1492-1421 (numérique)

Découvrir la revue

Citer cet article

Medhat-Lecocq, H. (2010). De la nécessité pour le traducteur de maîtriser les domaines spécialisés : le cas des systèmes juridictionnels. Meta, 55(2), 251-265. https://doi.org/10.7202/044238ar
Résumé de l'article

Depuis la fin du XIX ${ }^{\mathrm{e}}$ siècle, le droit égyptien, ayant comme principale source le Code Napoléon, est étroitement lié au droit français. S’appuyant sur ce principe, le traducteur d'un texte juridique, et plus spécialement dans le domaine juridictionnel, risque cependant de faire des confusions fâcheuses. Cela est dû au fait que les deux systèmes, égyptien et français, ont évolué différemment, chacun en fonction du contexte culturel et historique de leur pays respectif. L'objectif de la présente recherche est de montrer que toute traduction dans ce domaine doit impérativement se fonder sur une connaissance approfondie des deux systèmes. 


\title{
De la nécessité pour le traducteur de maîtriser les domaines spécialisés: le cas des systèmes juridictionnels
}

\author{
HÉBA MEDHAT-LECOCQ \\ Université Al-Azhar, Le Caire, Égypte \\ hebalecocq@yahoo.fr
}

\begin{abstract}
RÉSUMÉ
Depuis la fin $\mathrm{du} x \mathrm{xI}^{\mathrm{e}}$ siècle, le droit égyptien, ayant comme principale source le Code Napoléon, est étroitement lié au droit français. S'appuyant sur ce principe, le traducteur d'un texte juridique, et plus spécialement dans le domaine juridictionnel, risque cependant de faire des confusions fâcheuses. Cela est dû au fait que les deux systèmes, égyptien et français, ont évolué différemment, chacun en fonction du contexte culturel et historique de leur pays respectif. L'objectif de la présente recherche est de montrer que toute traduction dans ce domaine doit impérativement se fonder sur une connaissance approfondie des deux systèmes.
\end{abstract}

\begin{abstract}
Since the end of the $19^{\text {th }}$ century until now, the Egyptian law, whose principal source is the Code Napoléon, has been closely related to the French law. On this basis, the translator of legal texts, and particularly juridictional texts, is likeky to make infortunate confusions. A reason for this is that the Egyptian and French systems have evolved differently according to their specific cultural and historical contexts. The aim of this paper is to demonstrate that the translations in this field must absolutely be based on a deep knowledge of both systems.

MOTS-CLÉS/KEYWORDS

juridiction, Code Napoléon, pseudo-polysémie, hyperonymie, cooccurrence juridiction, Code Napoléon, pseudo-polysemy, hyperonymy, cooccurrence
\end{abstract}

\section{Introduction}

Point n'est besoin de rappeler l'importance de la langue juridique. Si elle concerne en premier lieu les juristes, elle intéresse également les traducteurs, les terminologues et, quoique résistant à toute vulgarisation, le grand public. Comme les autres langues spécialisées, celle du domaine juridique a ses propres caractéristiques. Son caractère figé et son refus de toutes sortes de reformulation la rendent, le plus souvent, inaccessible pour le lecteur profane. Il s'agit donc pour celui-ci d'un problème de compréhension du texte juridique. Qu'en est-il pour le traducteur spécialisé dans ce type de texte? Non seulement, il doit comprendre le message de l'émetteur, mais aussi le transmettre dans une autre langue à son récepteur.

De prime abord, le traducteur d'un texte spécialisé consultera son outil de prédilection: le dictionnaire spécialisé, dans le cas présent, un dictionnaire juridique. Mais, ne traduisant que des mots isolés, comme c'est le cas de la quasi-totalité des lexiques bilingues disponibles sur le marché, le dictionnaire juridique ne suffit pas, 
à lui seul, pour traduire l'ensemble du texte. Restant toujours sur sa faim, le traducteur aura recours aux documents authentiques dans la langue cible, démarche lui permettant, d'abord, une meilleure compréhension du domaine, et ensuite, la constitution de son propre lexique contextuel du domaine faisant l'objet de son étude.

Dans le meilleur des cas, cette méthode, bien qu'exigeant beaucoup de temps, doit garantir une traduction fidèle du texte de départ (TD). Par fidèle, nous entendons une fidélité «au sens et non pas à la langue» (Hurtado Albir 1990: 72). Or, l'expérience nous montre que les choses ne sont pas si simples que cela et que le traducteur, contraint par le facteur temps, se trouve face à un grand nombre de signes linguistiques et de notions qu'il a bien du mal à cerner et, par conséquent, à utiliser correctement tout en évitant les contresens. Ceci est plus particulièrement sensible dans le domaine juridictionnel où le vocabulaire lié aux différentes juridictions est très précis et ne peut être utilisé à mauvais escient, d'où notre proposition de présenter cette recherche qui a pour objectif principal de servir d'outil au traducteur d'un texte juridique se rapportant plus précisément au domaine en question.

Ainsi, nous présenterons une étude terminologique et traductologique des deux systèmes juridictionnels, français et égyptien. Pour ce faire, nous poserons dans un premier temps, le cadre de notre étude, tout d'abord en rappelant les rapports historiques entre les deux systèmes, puis en délimitant le discours juridictionnel par rapport aux autres types de discours juridiques. Dans un deuxième temps, il conviendra de comparer les deux systèmes quant à leur organisation et leur fonctionnement. Cette deuxième étape se révèle être particulièrement nécessaire du fait de l'apport extralinguistique important au discours juridictionnel. En effet, ce n'est qu'en établissant une comparaison entre les deux systèmes juridictionnels, que nous pourrons surmonter les difficultés de traduction des textes relevant de ce domaine de spécialisation.

Enfin, une fois les points communs et les dissimilitudes entre les deux systèmes cernés, nous pourrons mener l'étude traductologique proprement dite. Pour ce faire, nous tenterons de lever quelques ambiguïtés traductologiques et terminologiques naissant du passage d'une langue à l'autre ou, plus précisément, d'un système à l'autre. De plus, le caractère figé du discours en question exige, de la part du traducteur, une étude approfondie de la cooccurrence dans ce domaine. Nous terminons enfin cette approche traductologique par quelques remarques sur les caractéristiques du vocabulaire juridictionnel.

\section{Cadre de l'étude}

\subsection{Rapports historiques entre les droits français et égyptien}

Personne ne peut nier les rapports intimes existant entre le droit égyptien et le droit français. Pour trouver l'origine de ces rapports, il faut remonter à la fin du XIX $x^{e}$ siècle quand les Occidentaux vivant en Égypte jouissaient des droits et privilèges des Capitulations de Soliman le Magnifique ${ }^{1}$, d'où la création, dans ce pays, de nombreux tribunaux consulaires qui, adoptant plusieurs langues et plusieurs législations, créèrent un certain désordre dans la justice.

Pour mettre fin à cette confusion judiciaire, Nubar Pacha, ministre du Khédive Ismaill, demanda à Jacques H. P. Maunoury, un avocat français, de préparer une législation égyptienne dans l'esprit de celles des puissances européennes (Ghali 1983: 
320-329). De nationalité française, Maunoury recopia le Code Napoléon, tout en y introduisant, évidemment, quelques remaniements concernant le statut des personnes, qui devaient respecter les règles du droit islamique, et en l'adaptant aux us et coutumes du peuple égyptien (Attallah 1995: 21-22).

L'élaboration du Code mixte en 1875 entraîna la création des Tribunaux mixtes qui avaient pour rôle essentiel de connaître les litiges entre les citoyens égyptiens et les étrangers, et au sein desquels les juges locaux et les juges occidentaux travaillaient de concert. Afin d'éliminer les Tribunaux mixtes et pour remplacer le Code mixte par une législation égyptienne, le gouvernement d'Égypte désigna Édouard Lambert, un juriste français, et Sanhoury Pacha, un de ses anciens élèves, pour préparer en commun, dans le cadre d'un comité mixte, un code civil égyptien (Attallah 1995: 23). Ceci explique l'influence importante qu'a exercée le droit français sur la législation égyptienne, influence qui toucha d'abord le code civil pour s'étendre au code pénal et au code administratif.

\subsection{Le discours juridictionnel}

Le discours juridique est loin d'être uniforme. À la lumière de la typologie proposée par Cornu (1990: 216-222), il est possible de distinguer les discours législatif (lois, règlements, etc.), coutumier (maximes et adages du droit), conventionnel (contrats, traités et pactes, etc.) et juridictionnel. Même au sein de ce dernier, le discours varie d'un sous-domaine à l'autre, selon qu'il s'agisse des plaidoiries (avocats et représentants du parquet), des déclarations des témoins ou des décisions rendues (jugements et arrêts).

C'est ce dernier type de discours qui, du fait de sa complexité extrême et des problèmes traductologiques qu'il pose, nous intéresse le plus. Cependant, tout traducteur de décisions judiciaires, notamment s'il travaille avec le français et l'arabe, découvrira aussitôt qu'il est impossible d'aboutir sans comprendre le fonctionnement des deux systèmes juridictionnels qui, bien qu'émanant d'une seule source, le Code Napoléon, comportent beaucoup de différences. Une étude comparative des deux systèmes mérite donc d'être entreprise, de façon à fonder ultérieurement une étude sur la traduction des décisions judiciaires. Soulignons toutefois que tous les sousdomaines indiqués ci-dessus ont leurs spécificités linguistiques et mériteraient donc, chacun, une étude à part entière.

\section{Les systèmes juridictionnels français et égyptien}

\subsection{Approche comparative}

Il est évident que le système juridictionnel égyptien, inspiré de celui de la France, a beaucoup de points communs avec ce dernier. Au cours de son processus de création, un grand nombre de principes qui constituent la base fondamentale du système juridictionnel français ont été importés. Citons, à titre d'exemples, le principe de la dualité des juridictions (mahâkimu âdiyya wa mahâkimu idâriyya: 'juridictions judiciaires et administratives ${ }^{2}$ ), l'organisation hiérarchique des juridictions (premier degré, deuxième degré et juridiction suprême placée au sommet de la hiérarchie), la publicité de la justice, l'inamovibilité des juges et le principe de la légalité des peines. 
Cependant, les circonstances politiques et historiques dans les deux pays vont introduire certaines différences dans les systèmes juridictionnels respectifs. C'est ce que nous tenterons de montrer grâce à une comparaison de leurs juridictions administratives et civiles, d'une part, et de certaines juridictions situées en dehors de ces dernières, d'autre part. Loin d'être exhaustive, cette analyse se limitera aux juridictions principales, ainsi qu'à quelques juridictions et organes susceptibles de poser des problèmes sur le plan traductologique.

À première vue, la comparaison entre les juridictions administratives égyptiennes et françaises révèle une même structure hiérarchique. Nous trouvons dans les deux systèmes:

- des juridictions de premier degré: les instances en France (tribunaux administratifs) et en Égypte portent des noms équivalents (mahâkimu idâriyya: 'tribunaux administratifs');

- une juridiction de second degré: la Cour administrative d'appel en France correspond à la 'Cour du contentieux administratif' (mahkamatu l-qadâ'i l-idâri) en Égypte;

- une juridiction suprême: le Conseil d'État en France et la Cour suprême administrative (al-mahkamatu l-'idâriyyatu l-'ulyâ) en Égypte.

Personne ne peut donc contester l'idée selon laquelle le législateur égyptien, pour créer l'ordre administratif, s'est beaucoup inspiré de son équivalent français. Cependant, cette idée risque d'être fort trompeuse, surtout pour un traducteur profane ignorant le fonctionnement des juridictions administratives des deux pays et leurs compétences respectives.

Ainsi, le terme égyptien majlisu d-dawla, qui constitue une traduction littérale de Conseil d'État (utilisé en France pour désigner la juridiction administrative suprême), bien que fréquemment utilisé par les juristes égyptiens, ne figure pas parmi les juridictions administratives égyptiennes susmentionnées et ne désigne donc aucune juridiction effective. Une comparaison beaucoup plus minutieuse entre les deux institutions montre que le législateur égyptien n’a pas copié d'emblée la structure du Conseil d'État français dans son intégralité. Rappelons que ce dernier est divisé en six sections, cinq assurant une fonction administrative d'avis et une sixième, celle du contentieux, avec une fonction juridictionnelle (Van Lang, Gondouin et al. 2005: 83). En plus, dans sa fonction juridictionnelle, le Conseil d'État français est placé au sommet des juridictions administratives.

Le Conseil d'État égyptien (majlisu d-dawla), quant à lui, est divisé en trois sections: la section d'avis (qismu l-fatwâ), la section de législation (qismu t-tashrî‘) et la section du contentieux (al-qismu l-qadâ' $\mathbf{i}$ ). C'est sous cette dernière que sont regroupées les juridictions administratives égyptiennes. Par conséquent, contrairement au système français, le Conseil d'État (majlisu d-dawla) en Égypte n'est pas une juridiction, mais c'est au sein de cet organe, et plus précisément de la section du contentieux que nous trouvons toutes les juridictions administratives égyptiennes. Ainsi, le terme majlisu d-dawla est utilisé par quelques juristes égyptiens, tantôt pour désigner l'ensemble des juridictions administratives, avec pour synonyme almahâkimu l-idâriyya ('juridictions administratives'), tantôt tantôt pour désigner l'ensemble de l'ordre administratif (al-qadâ'u l-idâri) (Khafaga 1998: 15-17).

Cette incompatibilité entre les termes Conseil d'État (en France) et majlisu d-dawla (en Égypte), en raison des notions qu'ils véhiculent, vient probablement du fait qu'au moment de la création de ce dernier, en 1946, le législateur égyptien n’a 
prévu que la 'Cour du contentieux administratif' (mahkamatu l-qadâ'i l-idâri) comme seule juridiction administrative (loi 112) située à l'intérieur de la section du contentieux. Ensuite, pour faire face au grand nombre de procès en suspens et, s'inspirant du modèle juridictionnel français, il créa en 1954 (loi 47) les 'tribunaux administratifs' (al-mahâkimu l-'idâriyya) comme juridictions de premier degré, tout en confiant à la 'Cour du contentieux administratif' (mahkamatu l-qadâ'i l-idâri) les attributions d'une juridiction de deuxième degré pour quelques affaires. Enfin, en 1955 (loi 165), une juridiction suprême, équivalente à celle du Conseil d'État français, fut créée, mais sous le nom de al-mahkamatu l-idariyyatu 1-'ulyâ ('Cour suprême administrative') et non pas majlisu d-dawla. (Abdel Wahab et Osman 2000: 113-121).

L'Égypte s'écartera toutefois du modèle français par la création, en 1958 (loi 117), à l'intérieur de l'ordre administratif, des 'juridictions disciplinaires' (mahâkimu ta'dîbiyya), dont la compétence est de juger les fonctionnaires publics pour les affaires pécuniaires et disciplinaires. Signalons que ces juridictions ne correspondent pas à la Cour de discipline budgétaire et financière créée en France, celle-ci ayant pour fonction de sanctionner les ordonnateurs (les agents publics responsables de l'exécution d'un budget) en matières financière et budgétaire (Bissardon 2005 : 196).

Soulignons également que si la France est dotée de la Cour des comptes, l'Égypte connaît l' 'Organisme central des comptes' (al-jihâzu l-markaziyyu li l-muhâsabât). Cependant, ce dernier ne constitue pas une juridiction et ne rend pas de décisions. Sa compétence se limite au seul contrôle et, en cas d'infraction, il renvoie l'affaire à la juridiction administrative concernée. Il serait donc erroné de traduire al-jihâzu l-markaziyyu li 1-muhâsabât par Cour des comptes, solution à laquelle ont recours quelques traducteurs, dans un souci de clarifier le sens pour le destinataire français.

Les similitudes entre les juridictions judiciaires françaises et égyptiennes sont beaucoup plus importantes. D’abord, comme en France, le législateur égyptien distingue les 'juridictions civiles' (mahâkimu madaniyya) et les 'juridictions pénales' (mahâkimu jinâ'iyya). En Égypte, comme c'est le cas pour l'ordre administratif, la structure hiérarchique des juridictions judiciaires est semblable à celle de la France. Ainsi, en matière civile, les deux pays sont dotés de:

- juridictions de premier degré: les tribunaux de grande instance, en France, leurs homologues égyptiens s'appelant mahâkimu ibtidâ'iyya ou mahâkimu kulliyya; les tribunaux d'instance, adoptés par le législateur égyptien sous le nom de mahâkimu juz'iyya; les tribunaux de commerce, appelés mahâkimu t-tijâriyya; enfin, en France, les conseils de prud'hommes, compétents pour régler les litiges entre salariés et employeurs, sont comparables aux 'tribunaux des affaires des ouvriers' (mahâkim shu'ûni l-'ummâl) et ont les mêmes compétences;

- juridictions de second degré: les cours d'appel en France, les entités homologues égyptiennes s'appelant mahâkimu l-isti'nâf. Cependant, en plus de ces dernières juridictions, l'Égypte a confié aux 'tribunaux de grande instance' (al-mahâkimu l-ibtidâ'iyya ou al-mahâkimu l-kulliyya) les appels formés contre les jugements rendus par les 'tribunaux d'instance' (al-mahâkimu l-juz'iyya) et les ordonnances rendues par le 'juge des référés' (qâdi l'umûri l-musta'jala);

- une juridiction suprême: la Cour de cassation, adoptée en Égypte sous le nom de mahkamatu n-naqd.

Comme nous venons de le voir, l'Égypte ne se distingue de la France que par ses juridictions de second degré, les compétences d'appel étant réparties entre ce qui 
correspond aux cours d'appel (mahâkimu l-isti'nâf) et l'équivalent des tribunaux de grande instance (mahâkimu ibtidâ'iyya ou mahâkimu kulliyya). Ceci explique l'utilisation, par les juristes égyptiens, de deux synonymes pour désigner ces tribunaux. Le terme mahâkimu ibtidâ'iyya ('juridictions de premier ressort') constitue le plus ancien des deux. Mais, selon Zaghloul (1998: 410), quelques juristes égyptiens, constatant que ce terme n'évoque que partiellement les compétences attribuées aux juridictions en question, le remplacèrent par mahâkimu kulliyya ('juridictions globales'), puisqu'elles statuent autant en premier qu'en dernier ressort en tant que juridictions d'appels. Ce dernier terme, bien que de plus en plus fréquent, n'a pas réussi à évincer mahâkimu ibtidâ'iyya, qui continue à être utilisé par un grand nombre de spécialistes du domaine. C'est pour cette raison que nous les gardons tous les deux comme synonymes concurrents.

En matière pénale, le système judiciaire égyptien s'est fondé non seulement sur la même structure hiérarchique que celle de la France, mais aussi sur le même principe de la division des infractions en contraventions, délits et crimes. On note peu de différences dans la conception de certaines juridictions et donc, dans leurs compétences. Ainsi, les deux ordres pénaux connaissent:

- des juridictions de premier degré: en France, il existe des tribunaux de police pour les contraventions, des tribunaux correctionnels pour les délits et des cours d'assises pour les crimes. Par contre, en Égypte, un seul type de juridiction est compétent pour les contraventions et les délits: il s'agit des 'tribunaux correctionnels des délits et contraventions' (mahâkimu l-junahi wa l-mukhâlafât) et un autre pour juger les crimes, les 'cours d'assises' (mahâkimu l-jinâyât);

- des juridictions de second degré: la France a les cours d'appel, compétentes pour les appels formés contre certains jugements rendus par les tribunaux de police (d'autres jugements ne peuvent faire l'objet d'appels) et par les tribunaux correctionnels; les cours d'assises en formation d'appel statuant sur les appels formés contre les décisions rendues par les cours d'assises; l'Égypte a l'équivalent des tribunaux de grande instance (mahâkimu kulliya ou mahâkimu ibtidâ'iyya) pour les appels formés contre les jugements rendus par les 'tribunaux correctionnels des délits et des contraventions' (mahâkimu l-junahi wa l-mukhâlafât). Quant aux arrêts rendus par les 'cours d'assises' (mahâkimu l-jinâyât), ils ne peuvent faire l'objet d'appels;

- une juridiction suprême: en France comme en Égypte, une seule juridiction peut être saisie des pourvois en cassation formés contre les arrêts et les jugements rendus en premier et dernier ressort. Il s'agit de la Cour de cassation, adoptée en Égypte sous le nom de mahkamatu n-naqd.

Une analyse fine des différences existant entre les deux ordres pénaux montre que le législateur égyptien, contrairement à son homologue français, a réuni les deux juridictions connaissant les délits et les contraventions en une seule: le 'tribunal correctionnel des délits et contraventions' (mahkamatu l-junahi wa l-mukhâlafât), tribunal qu'il a mis en place au sein du 'tribunal d'instance' (al-mahkamatu l-juz'iyya). Il a donc trouvé logique que les appels formés contre les jugements du 'tribunal correctionnel des délits et des contraventions' soient confiés au al-mahkamatu l-kulliyya (ou al-mahkamatu l-ibtidâ'iyya), situé au-dessus du 'tribunal d'instance'.

Par contre, pour le législateur français, la Cour d'appel constitue la seule juridiction de deuxième degré. C'est la raison pour laquelle il a confié à cette juridiction, et plus spécialement à une chambre des appels correctionnels à l'intérieur de celle-ci, 
les appels formés contre les jugements rendus par le tribunal correctionnel ainsi que ceux, susceptibles d'appel, rendus par le tribunal de police.

Quant aux 'cours d'assises' égyptiennes (mahâkimu l-jinâyât), elles suivent l'ancien modèle français selon lequel leurs décisions ne sont pas susceptibles d'appel devant une cour d'appel, mais peuvent faire l'objet de cassation. Or, conformément à une loi récente en France ${ }^{3}$, les décisions rendues par les cours d'assises sont frappées d'appel devant d'autres cours d'assises en formation d'appel. Mais l'Égypte n'a pas suivi la France dans cette évolution.

Citons enfin quelques juridictions classées en dehors des deux ordres, administratif et judiciaire, et qui prouvent, une fois de plus, que bien que liés par des rapports étroits, les deux systèmes évoluent différemment, chacun selon son contexte local. Si le législateur français a créé le Conseil constitutionnel chargé, entre autres, de contrôler la constitutionnalité des lois et de veiller à la régularité des élections présidentielles et législatives (Guillien et Vincent 1985), le système égyptien est doté d'une 'Haute cour constitutionnelle' (al-mahkamatu d-dustûriyyatu l-'ulyâ). Comme le Conseil constitutionnel français, cette juridiction est habilitée à contrôler la constitutionnalité des lois et des règlements et à surveiller la régularité des élections. Par contre, en plus de ces attributions, elle connaît des conflits de compétence entre les deux ordres, administratif et judiciaire, alors qu'en France ces conflits sont confiés à un Tribunal des conflits créé à cet effet, " composé à parts égales de magistrats des deux ordres et présidé par le ministre de la justice, garde des Sceaux» (Aubert 2002: 126).

En Égypte, il convient toutefois de souligner que les conflits de compétence ont d'abord été confiés, en 1949, à la 'Cour de cassation' (mahkamatu n-naqd). Cependant, cette décision a fait l'objet de beaucoup de critiques, étant donné que cette dernière est à la tête des juridictions judiciaires et, par conséquent, ne peut être considérée comme neutre pour trancher les conflits de compétence entre les deux ordres (Naguib 1998: 68-69). Le législateur égyptien a donc eu recours au modèle français pour créer, en 1959, un 'Tribunal des conflits' (mahkamatu t-tanâzu'). Composé de trois magistrats de la 'Haute cour administrative' (al-mahkamatu l-idâriyyatu l-'ulyâ) et de trois autres de la 'Cour de cassation' (mahkamatu n-naqd), mais présidé par le président de cette dernière juridiction, le 'Tribunal des conflits', jugé à son tour favorable à l'ordre judiciaire, n’a pas tardé à faire l'objet de contestations. Les conflits de compétence ont été finalement attribués, en 1969, à la 'Cour suprême' (al-mahkamtu l-'ulyâ), devenue, en 1979, la 'Haute cour constitutionnelle' (al-mahkamatu d-dusturiyyatu l-'ulyâ) (Khafaga 1998: 9).

Ainsi, tout au long de son histoire, le système juridictionnel égyptien n'a pas cessé de puiser dans son équivalent français, mais les impératifs culturels et historiques de chacun des deux pays ont joué un rôle primordial dans la conception et l'évolution de leurs schémas juridictionnels respectifs.

\subsection{Approche traductologique}

Une fois le fonctionnement des deux systèmes juridictionnels élucidé, ce rapport «ambigu» et «chaotique» (Gémar 1995: 10) entre la langue et le droit commence à disparaître progressivement. C'est à ce moment-là que le traducteur se permet de constituer son propre lexique et de tirer certaines conclusions sur les plans traductologiques et terminologiques. 


\subsubsection{Traduction par correspondances et traduction par équivalences}

Reprenons maintenant les deux termes, Conseil d'État, et majlisu d-dawla, qui en constitue la traduction littérale. Nous avons vu précédemment (section 3.1.) que, bien qu'ils soient cohérents quant à certains traits notionnels, ils se distinguent par le fait que le premier constitue en France une juridiction, contrairement au second en Égypte, bien que ce dernier réunisse l'ensemble des juridictions administratives. Le traducteur invité à traduire la phrase suivante:

(1a) Le Conseil d'État français a rejeté le pourvoi formé contre l'arrêt rendu par la Cour administrative d'appel.

proposera probablement:

(1b) rafada majlisu d-dawlati l-firinsiyyu tána l-hukmi s-sâderi min mahkamati listi'nâfi l-idâriyya.

[litt.: Le Conseil d'État français a rejeté le pourvoi formé contre l'arrêt rendu par la Cour administrative d'appel.]

Le texte d'arrivée (TA) est obtenu grâce à une traduction par correspondance, c'est-à-dire qui fait usage des «correspondances lexicales et syntaxiques préexistantes entre les deux langues» (Lederer 2002: 18). Bien qu'irréprochable, cette traduction reste imprécise, puisqu'elle n'a pas «[produit] chez le lecteur de la LA [langue d'arrivée] le même effet généré chez celui de la LD [langue de départ]» (Sparer 1979: 68). Le premier, égyptien, éventuellement non initié au système français et imprégné du contexte culturel juridique de l'Égypte, penserait à une cour suprême placée à l'intérieur du Conseil d'État français, omise par l'auteur du TD ou le traducteur et remplacée par son superordonné partitif majlisu d-dawla (Conseil d'État), puisque, rappelons-le, le 'Conseil d'État' en Egypte n'est pas une juridiction, mais intègre toutes les juridictions administratives et à leur tête 'la Cour suprême administrative' (al-mahkamatu 1-'idariayyatu l-'ulyâ).

Pour lever cette équivoque, le traducteur doit informer son destinataire de la fonction précise du Conseil d'État français, quitte à ajouter une idée ne figurant pas explicitement dans le TD, en proposant, par exemple, pour cette même phrase la traduction suivante:

(1c) rafada majlisu d-dawlati l-firinsiyyu, wa howa yu 'âdilu l-mahkamata l-'idariayyata l-'ulyâ bi-Misr, ta'na l-hukmi s-sâderi min mahkamati l-isti'nâfi l-'idâriyya.

[litt.: Le Conseil d'Etat français, qui constitue l'entité française équivalente à 'la Cour suprême administrative' en Egypte, a rendu un arrêt de rejet contre le pourvoi en cassation déposé contre l'arrêt de la Cour du contentieux administratif.]

Cette deuxième traduction ne se distingue de la précédente que par la phrase intercalée wa howa yu 'âdilu l-mahkamata l-'idariayyata l-'ulyâ bi-Misr (qui constitue l'entité française équivalente à la cour suprême administrative en Égypte). Il s'agit donc ici d'une traduction par équivalences, puisque le traducteur «[s'est construit] une image mentale de la situation et, ayant trouvé "le sens" du texte, il l'exprime par équivalences, de façon idiomatique en langue d'arrivée» (Sparer 1979: 18).

La même équivoque peut se produire dans l'interprétation d'un texte sur la juridiction égyptienne statuant sur les conflits de compétence entre les deux ordres. Essayons de traduire la phrase suivante: 
(2a) wa qad qâmat al-mahkamatu l-mukhtassatu bi- tanâzu'il-wilâyati bi-halli hadhihi l-mushkilati wa bi-ta'yîni l-qadâ'i l- 'idâriyyi li l-fasli fi hadhihi l-qadiyya.

(2b) La juridiction connaissant les conflits de compétence a résolu ce problème et a désigné l'ordre administratif pour statuer sur cette affaire.

Nous remarquons dans cette phrase, ainsi que sa traduction par correspondances, que l'auteur du TD n'a pas précisé le nom de la juridiction en question et s'est contenté d'en donner une glose (al-mahkamatu l-mukhtassatu bi- tanâzu'i lwilâyati - 'la juridiction connaissant des conflits de compétence').

Dans ce cas, le risque est double. Tout d'abord, le destinataire français du TA, ne sachant pas que cette juridiction peut être en Égypte la 'Cour de cassation' (mahkamatu n-naqd) entre 1949 et 1958, le 'Tribunal des conflits' (mahkamatu t-tanâzu') entre1959 et 1969, la 'Cour suprême’ (al-mahkamatu l-'ulyâ) de 1969 à 1978 ou la 'Haute cour constitutionnelle' (al-mahkamatu l-dustûriyyatu l-'ulyâ) à partir de 1979 (section 3.1.), risque de penser directement au 'Tribunal des conflits' (mahkamatu t-tanâzu'), seule juridiction, selon sa propre culture, compétente pour trancher ce genre de conflits.

$\mathrm{Si}$, par contre, le traducteur décide de traduire la phrase en question par équivalences, mais ignorant ou ne cherchant pas à connaître l'historique de ce type de juridiction en Égypte, il risque lui aussi de penser directement au 'Tribunal des conflits', d'autant plus que beaucoup de dictionnaires bilingues (français/arabe) citent ce dernier comme équivalent de mahkamatu t-tanâzu' sans toutefois évoquer les réformes introduites au fil des années sur les conflits de compétences (Hakki 1979).

Donc, la traduction d'une telle phrase exige du traducteur, d'abord une formation préalable dans le domaine concerné, ensuite une lecture paratextuelle lui permettant de repérer la date de production du TD afin de désigner la juridiction citée par l'auteur et, enfin, une fois ces deux étapes franchies, une traduction par équivalences tenant compte de l'implicite caché derrière les signes et offrant ainsi au destinataire du TA et avec le même degré de transparence, le message reçu par celui du TD.

Imaginons que ce texte soit publié en 2000. Dans ce cas-là, la juridiction concernée sera la 'Haute cour constitutionnelle' (al-mahkamatu l-dustûriyyatu l-'ulyâ). Nous proposons donc comme traduction de l'exemple (2a):

(2c) La Haute cour constitutionnelle, compétente pour trancher les conflits de compétence, a résolu ce problème en faveur de l'ordre administratif qui devra statuer sur cette affaire.

En ajoutant Haute cour constitutionnelle (qui correspond à al-mahkamatu l-dustûriyyatu 1-'ulyâ, lequel n'est pas explicité dans le TD) et en précisant au destinataire du TA que cette juridiction est compétente en Égypte pour trancher les conflits de compétence, nous avons eu recours à une traduction par équivalences, avec pour objectif de rendre hommage au TD.

Toutefois, les deux exemples précédents ne doivent pas nous imposer la traduction par équivalences comme seule solution devant le traducteur. C’est le contexte qui oblige ce dernier à faire le choix entre celle-ci et la traduction par correspondances. Si dans le premier exemple l'auteur du TD avait évoqué le Conseil d'État dans le contexte suivant:

(3a) Le Conseil d'État français est divisé en six sections. 
une traduction par correspondances aurait suffi pour passer le message complet au destinataire du TA, puisqu'il s'agit de la première notion de Conseil d'État, celle de l'organe administratif de l'État, qui existe dans les deux cultures. Le traducteur peut donc proposer:

(3b) yanqasimu majlisu d-dawlati l-firansiyyu'ilâ sittati 'aqsâm.

[litt.: Le Conseil d'État français est divisé en six sections.]

en ayant ainsi recours à un simple transcodage du TD.

Donc, tout texte traduit sera constitué forcément d'un mélange des deux types de traductions. C'est en fonction du contexte de la LD que le traducteur doit choisir entre les deux démarches afin de garantir une traduction fidèle "au sens et non pas à la langue» (Hurtado Albir 1990: 72).

\subsubsection{Désambiguïsation terminologique}

Jusque-là, notre analyse a été axée sur les termes, français et arabes, utilisés pour désigner les différentes juridictions dans les deux pays. Mais à côté de ces termes, il $\mathrm{y}$ a tout un vocabulaire se rapportant à ces juridictions et qui mérite d'être analysé et bien assimilé par le traducteur. Pour commencer cette analyse, il faut souligner que le législateur égyptien, bien que puisant dans le droit français, a refusé dès le début tout emprunt à la langue française, décision logique, puisque, rappelons-le, une des raisons principales de la création de la législation égyptienne était de mettre fin aux problèmes linguistiques naissant de l'utilisation de plusieurs langues à l'intérieur des tribunaux consulaires (section 2.1.). L’Égypte a donc importé de la France des notions appartenant au domaine concerné, et non pas les termes les désignant. Une fois transposées en Égypte, ces notions devaient toutefois mobiliser l'idiomaticité de la nouvelle langue et à faire appel aux termes égyptiens pertinents.

Prenons l'exemple du terme mahkama, utilisé en tant que vecteur d'une seule notion, celle de toute juridiction, quels que soient son degré et sa compétence. En français, on trouvera une notion générique véhiculée par le terme juridiction, celle-ci embrassant deux autres notions spécifiques: 1) l'une, désignée par tribunal, pour les juridictions du premier degré; 2) l'autre, cour, pour les juridictions du second degré (les cours d'appel et la Cour administrative d'appel), la Cour de cassation et quelques autres dont les décisions ne sont pas frappées d'appel devant une cour d'appel, mais devant d'autres cours. C'est le cas des cours d'assises dont les arrêts sont susceptibles d'appel devant une cour d'assises en formation d'appel, ainsi que de la Cour des comptes et de la Cour de discipline budgétaire et financière, dont les décisions font l'objet de cassations directement devant la Cour de cassation. Nous avons donc trois termes français (juridiction, tribunal et cour) pour un seul arabe terme, mahkama. Cela est dû à la différence de conceptualisation des différentes notions au sein des deux systèmes, français et égyptien.

La même remarque s'applique au terme hukm. Alors que ce terme égyptien renvoie à tout type de décision juridictionnelle, le français utilise le terme générique décision, qui va embrasser deux autres notions spécifiques: 1) la décision des juridictions du premier degré, désignée par jugement; 2) la décision rendue par une cour (cours d'appel, d'assises, de cassation, etc.) ou par le Conseil d'État, désignée par arrêt. Le terme arabe hukm unique aura donc pour équivalents français trois termes distincts (décision, jugement et arrêt). Ce phénomène de pseudo-polysémie ${ }^{4}$ (Medhat- 
Lecocq 1997: 1639-1640) caractérisant quelques termes arabes appartenant au domaine juridique est susceptible de créer, chez le traducteur peu initié, ce que Gérard Cornu appelle une amphibologie. Selon ce dernier,

l’amphibologie ou ambiguité est un accident de la polysémie. Elle se produit lorsque, dans un texte, un terme polysémique est utilisé par le locuteur de telle manière qu'il crée pour le récepteur l'équivoque, c'est-à-dire le doute et l'hésitation sur celui des sens dans lequel il faut alors prendre le terme (Cornu 1990: 90).

Toujours concernant les décisions des différentes juridictions, le traducteur doit connaître et utiliser très précisément les termes et expressions appropriés aux juridictions de chaque degré, du fait qu'ils résistent à toute forme de synonymie ou de reformulation, une particularité générale de la langue juridique.

Pour les tribunaux du premier degré:

- les parties en litige sont le demandeur / la demanderesse (al-mudda'i / al-mudda'iya) et le défendeur / la défenderesse (al-mudda'a 'alayhi / al-mudda'a 'alayha);

- concernant les décisions, le tribunal du premier degré rend un jugement (tasduru hukman); si le jugement répond positivement à la demande, donc le tribunal accueille la demande ou fait droit à la demande (qabilat ad-da'wa); si c'est le contraire, le tribunal déboute la demande ou le demandeur est débouté (rafadat ad-da'wa).

Pour les juridictions d'appel:

- les parties en litige sont celles qui forment l'appel, l'appelant (al-musta'nif), qui fait appel ou interjette appel (yasta'nif) et celles contre qui l'appel est formé, l'intimé (al-musta'naf diddahu);

- concernant les décisions, une cour d'appel rend un arrêt (tasduru hukman); soit elle rend un arrêt confirmatif (tu'ayyidu l-hukma s-sâbiq), soit elle rend un arrêt infirmatif (tarfudu l-hukma s-sâbiq).

Pour la cour de cassation:

- les parties en litige sont le demandeur au pourvoi (al-tấin) qui se pourvoit, se pourvoit en cassation ou forme un pourvoi en cassation (yat'anu bi l-naqd) et le défendeur au pourvoi (al-mat' $\mathbf{u}$ u diddahu);

- concernant les décisions, la cour de cassation rend un arrêt (tasduru hukman); soit elle rend un arrêt de rejet (tarfudu t-ta'n), soit elle rend un arrêt de cassation (ou de renvoi) (tanqudu al-hukm).

Une tentative de la part du traducteur de remplacer ces expressions par d'autres qu'il juge synonymes ou plus accessibles (en remplaçant, par exemple, le tribunal déboute la demande par le tribunal refuse la demande) ne peut conduire qu'à l'échec et sa traduction sera jugée imprécise.

La dernière remarque sur le vocabulaire des décisions concerne le ressort des juridictions. En effet, les différentes juridictions, selon leur degré et leur compétence, statuent:

- en premier ressort (hukmun 'ibtidâ'iyyun) ou à charge d'appel (hukmun qâbilun li 1-isti'nâf), lorsque le jugement est susceptible d'appel devant une juridiction plus importante;

- en premier et dernier ressort (hukmun 'ibtidâ'iyyun wa nihâ'iyyun) quand la juridiction concernée est la première à être saisie pour cette affaire avec impossibilité d'interjeter appel, un recours en cassation restant toujours possible;

- en dernier ressort (hukmun nihâ'iyyun) lorsque la juridiction est saisie pour un recours contre une décision rendue en premier ressort. 


\subsection{3. Étude de la cooccurrence}

Les cooccurrents d'un terme sont les mots qui accompagnent, de façon plus ou moins figée, ce terme dans les différents contextes. Dans le domaine en question, la cooccurrence joue un rôle très important du fait que la langue juridique, et plus particulièrement la langue juridictionnelle, a un caractère figé et refuse toute sorte de reformulation.

Parmi les nombreux cooccurrents à étudier dans ce sous-domaine juridique, nous pouvons proposer ceux concernant les juridictions (tribunal, cour, etc.) et les décisions.

À propos des juridictions:

- saisir le tribunal $(\mathrm{V}+$ Dét $+\mathrm{N})$ correspond à rafa'a ad-da'wata amâma l-mahkama $(\mathrm{V}+$ Dét $+\mathrm{N}+\mathrm{Adv}+$ Dét $+\mathrm{N})$;

- assigner quelqu'un devant le tribunal $(\mathrm{V}+\mathrm{N}+\mathrm{Adv}+$ Dét $+\mathrm{N})$ correspond à istad'a fulânan amâma l-mahkama $(\mathrm{V}+\mathrm{N}+\mathrm{Adv}+\mathrm{Dét}+\mathrm{N})$;

- statuer sur (V + Prép) correspond à tafsilu fi (V + Prép); par exemple: cette juridiction statue sur les litiges entre salariés et employeurs se traduit par hadhihi al-mahkamatu tafsilu fi al-monâza'âti bayna l'âmilîna wa arbâbi l-'amal;

- être compétent pour (V + Adj + Prép) correspond à min ikhtisâsiha (Prép + N + Dét) ou takhtassu bi $(\mathrm{V}+$ Prép); par exemple: ce tribunal est compétent pour les litiges entre commerçants se traduit par hadhihi al-mahkamatu takhtassu bi l-munâza'âti bayna t-tujjâr;

- être habilité à (V + Adj + Prép) correspond à min ikhtisâsiha (Prép + N + Dét) ou takhtassu bi (V + Prép); par exemple: la cour d'assises est habilitée à juger les crimes se traduit par takhtassu mahkamatu l-jinâyâti bi l-hukmi 'ala l-jarâ'im;

- connaître $(\mathrm{V})$ correspond à min ikhtisâsiha (Prép + N + Dét) ou takhtassu bi (V + Prép); par exemple: le tribunal correctionnel connaît des délits se traduit par takhatassu mahkamatu l- junahi bi l- fasli fi l-junah.

Concernant les décisions:

- rendre une décision $(\mathrm{V}+$ Dét $+\mathrm{N})$ correspond à yasduru hukman $(\mathrm{V}+\mathrm{N})$;

- susceptible de (Adj + Prép) correspond à qâbilun li (Adj + Prép); par exemple: un jugement susceptible d'appel se traduit par hukmun qâbilun li l-'isti'nâf;

- faire l'objet de (V + Dét + N + Prép) correspond à qâbilun li (Adj + Prép); par exemple: un arrêt qui fait l'objet de cassation se traduit par hukmun qâbilun li t-ta'ni bi n-naqd.

Nous voyons ainsi que le caractère figé de la langue juridique donne à la cooccurrence une importance primordiale. Pour le juriste français, le tribunal ne donne pas de jugement, il n'en émet pas non plus, mais il rend un jugement. Même cas pour le spécialiste égyptien qui utilise un seul verbe asdara (tasduru l-mahkamatu hukman). Le recours à un autre verbe dans un objectif de reformulation ne sera pas accepté dans ce domaine.

\subsubsection{Quelques caractéristiques du vocabulaire juridictionnel}

\subsubsection{La dérivation}

Comme toute langue spécialisée, celle du domaine juridictionnel constitue «une langue naturelle considérée en tant que vecteur de connaissances spécialisées » (Lerat 1995: 20). Elle a, par conséquent, les caractéristiques de la langue naturelle. Une des 
spécificités de cette langue est la formation des termes par dérivation. La dérivation, en français, peut se faire par:

- substantivation du participe présent appel $\rightarrow$ appelant

- substantivation du participe passé

intimer $\rightarrow$ intimé

assigner $\rightarrow$ assigné

accuser $\rightarrow$ accusé

- suffixation en -eur pour le masculin et en -esse pour le féminin demander $\rightarrow$ demandeur, demanderesse défendre $\rightarrow$ défendeur, défenderesse

- suffixation en -ation casser $\rightarrow$ cassation

La dérivation dans le vocabulaire juridictionnel égyptien, tout comme en arabe général, se fait par le biais des schèmes arabes de dérivation et l'emploi de quelques adverbes ou prépositions (diddahu, 'alayhi), par exemple:

- idda'a (verbe) $\rightarrow$ al-mudda'i (le demandeur) / al-mudda'a 'alayhi (le défendeur)

- ista'nafa (verbe) $\rightarrow$ al-musta'nifu (l'appelant) / al-musta'nafu diddahu (l'intimé)

- ta'ana (verbe) $\rightarrow$ at-tâ'inu (le demandeur au pourvoi) / al-mat' ûnu diddahu (le défendeur au pourvoi)

\subsubsection{L'hyperonymie logique}

Contrairement à l'arabe spécialisé du domaine juridictionnel, le français est caractérisé par une relation d'hyperonymie logique entre certains termes. Il s'agit d'une relation qui lie un hyperonyme (associé à une notion générique) avec ses hyponymes (associé à des notions spécifiques), comme on l'a vu plus haut avec les termes décision et juridiction.

\section{Conclusion}

S’il est évident qu'il existe un fort lien de parenté entre le droit égyptien et le droit français et, plus particulièrement, entre les juridictions des deux pays, tant sur le plan de leur organisation que de leur fonctionnement, force est de constater néanmoins la présence de différences non négligeables entre les deux systèmes juridictionnels. Par conséquent, le traducteur non initié, ne connaissant pas le fonctionnement des juridictions égyptiennes et françaises, peut avoir des difficultés de compréhension et risque, par la suite, de faire des contresens. Nous mettons donc l'accent sur l'importance des connaissances extralinguistiques qui sont de plus en plus exigées du traducteur spécialisé. À ce sujet, Houbert nous signale que les annonces d'emploi à l'intention des traducteurs dans les grands cabinets d'avocats internationaux stipulent que «les candidats doivent, pour que leur candidature soit seulement prise en compte, avoir suivi une formation juridique à part entière et être titulaires d'un diplôme de droit» (Houbert 2001: 10).

Il est donc indispensable pour tout traducteur dans ce domaine, voire dans tout autre domaine spécialisé, d'allier ses connaissances linguistiques et extralinguistiques. Ce n'est qu'en franchissant cette étape qu'il peut aboutir à une traduction fidèle au TD. Pour garantir cette fidélité «au sens et non pas à la langue» ou, autrement dit, 
pour produire une traduction interprétative et non pas une traduction de la langue, le traducteur doit tenir compte de trois éléments: premièrement, le «vouloir dire» de l'auteur du TD (Lederer 2006: 67), ce qui implique de traduire, non seulement l'explicite, mais aussi l'implicite caché derrière les signes, implicite sans lequel le message risque d'arriver tronqué, voire déformé, au destinataire du TA; deuxièmement, la barrière culturelle entre les deux langues, barrière qu'il ne cesse de franchir tout au long du processus de la traduction, faisant ainsi la navette entre les deux cultures afin d'éviter les pièges du contresens; quant au troisième élément, c'est celui de l'idiomaticité de la LA, ou, pour être moins lapidaire, les moyens d'expressions propres à cette langue et plus spécialement, dans le domaine concerné, les termes, les expressions et les cooccurrents, utilisés d'une manière plus ou moins figée par les spécialistes du domaine, et refusant toute sorte de reformulation. Il y a lieu de signaler, à cet effet, l'absence de dictionnaires bilingues contextuels qui tiennent compte de la cooccurrence dans les deux langues.

Cette étude ne constitue qu'une première étape des recherches dans le domaine juridictionnel, afin de fournir au traducteur tous les éléments lui permettant de traduire les décisions judiciaires.

\section{NOTES}

1. Selon les droits et privilèges des Capitulations de Soliman le Magnifique, tout Occidental installé dans un pays de l'Empire ottoman, dont l'Égypte, est jugé selon sa propre loi nationale dans un tribunal consulaire (dépendant de son consulat).

2. Les gloses (traductions littérales) des termes arabes ont été distinguées dans tout le texte, par rapport aux dénominations françaises, par l'usage de guillemets simples.

3. Avec la loi du 15 juin 2000 qui a permis une procédure d'appel contre les arrêts de la cour d'assises (Aubert 2002: 159-160).

4. Expression que nous avons proposée pour désigner la polysémie d'un terme, non pas dans sa propre langue, mais par rapport à une autre langue.

\section{RÉFÉRENCES}

Abdel Wahab, Mohamed Refaat et Osman, Mohamed Osman (2000): Al-qadâ’u l-idâri. Le Caire: Dar il-matbû'âti l-gâmi'iyya.

Attallah, Borham (1995): Les liens entre les droits égyptien et français. In: Les liens entre les droits égyptien et français - Études présentées à la troisième conférence des ministres francophones de la justice. Ministère de la justice R.A.E. :21-24.

Aubert, Jean-Luc (2002): Introduction au droit et thèmes fondamentaux du droit civil. Paris: Armand Colin.

Bissardon, Sébastien (2005): Guide de la langue juridique. Vocabulaire, pièges et difficultés. Paris: Litec.

Connu, Gérard (1990): Linguistique juridique. Paris: Montchrestien.

GÉMAR, Jean Claude (1995): Traduire ou l'art d'interpréter: fonctions, statut et esthétique de la traduction. Tome 1. Principes: Presses de l'Université du Québec.

Ghali, Mirrit Boutros (1983): Mémoires de Nubar Pacha. Beyrouth: Librairie du Liban.

Guillien, Raymond et Vincent, Jean (1985): Lexique des termes juridiques. Paris: Dalloz.

HАкKI, Mamdouh (1979): Dictionnaires des termes juridiques et commerciaux (français-arabe). Beyrouth: Librairie du Liban.

Houbert, Frédéric (2001): Existe-t-il des traducteurs spécialisés? Traduire. 187:3-10.

Hurtado Albir, Amparo (1990): La notion de fidélité en traduction. Paris: Didier Érudition.

Khafaga, Ahmed Refaat (1998): At-tanzîmu l-qadâ’iyyu fi gomhûriyyati misra l-'arabiyya. 1-25.

Al-markazu l-qawmiyyu li-l-dirâsâti l-qadâ’iyya (Centre National des Etudes juridiques au Caire). 
LEDERER, Marianne (2002): Correspondances et équivalences, faits de langue et faits de discours en traduction. In: Fortunato IsRAËL, dir. Identité, altérité, équivalence? La traduction comme relation. (Colloque international en hommage à Marianne Lederer, Paris, 24-26 mai 2000). Paris/Caen: Lettres modernes/Minard.

LEDERER, Marianne (2006): La traduction aujourd'hui: le modèle interprétatif. Caen: Lettres modernes Minard.

Lerat, Pierre (1995): Les langues spécialisées. Paris: PUF.

Medhat-Lecoce, Héba (1997): L’architecture islamique en Égypte. Elaboration d'une terminologie et problématique de sa traduction. Thèse de doctorat. Le Caire: Université Al-Azhar.

Naguib, Mohamed Fathi (1998): At- tanzîmu l-qadâ'iyyu l-masri. Le Caire: Daru n-nahdati al-'arabiyya.

SPARER, Michel (1979) : Pour une dimension culturelle de la traduction juridique. Meta. 24(1):6894.

Van Lang, Agathe, Gondouin, Geneviève et Inserguet-Brisset, Véronique (2005): Dictionnaire de droit administratif. Paris: Dalloz.

ZAGHLOUL, Ahmed Maher (1998): Usûl wa qawâ'ed al morâfa'ât wefqan limajmûati al-murâfa'âti al-madaniyyati wat-tujâriyyati wa t-tashrî̀ âti al-mukammelati laha wal-murtabitati biha. Le Caire: Daru an-nahdati al-'arabiyya Al-Qâhira. 